\title{
PHASE TRANSITION IN $\mathrm{O}_{2}$
}

\author{
KLAUS THOMSEN \\ I mindet om Uffe Haagerup
}

\section{INTRODUCTION}

The Cuntz algebra $\mathrm{O}_{2}$ is the universal $C^{*}$-algebra generated by a pair of isometries $V_{0}, V_{1}$ such that $V_{0} V_{0}^{*}+V_{1} V_{1}^{*}=1,\left[\mathrm{C}\right.$. Let $A=A^{*} \in O_{2}$. For each $t \in \mathbb{R}$ the universal property of $\mathrm{O}_{2}$ guarantees the existence of an endomorphism $\sigma_{t}^{A}$ of $\mathrm{O}_{2}$ such that

$$
\sigma_{t}^{A}\left(V_{i}\right)=e^{i t A} V_{i}, i=0,1 .
$$

Under appropriate assumptions on $A$ the family $\sigma_{t}^{A}, t \in \mathbb{R}$, constitutes a continuous one-parameter family of automorphisms of $\mathrm{O}_{2}$, and it is an interesting problem to determine the KMS-states for $\sigma^{A}$. The main purpose with the present note is to exhibit an example of such an action where the structure of KMS states is rich with a relatively dramatic phase transition, involving an abrupt passage at a certain critical temperature from uncountably many extremal KMS states to one and then none. That this can occur in $\mathrm{O}_{2}$ is perhaps surprising since up to now the one-parameter actions on $\mathrm{O}_{2}$, or any simple unital Cuntz-Krieger algebra for that matter, for which it has been possible to determine the structure of KMS states have all had a unique KMS state, cf. $\mathrm{OP},[\mathrm{E}]$. In view of the work of Exel, $[\mathrm{E}$, it is clear that the possibility of having a richer structure has to do with the failure of the Ruelle-Perron-Frobenius theorem for certain potential functions; in particular, for the function $F$ below. In this respect, as well as others, the present work is related to the work of Hofbauer, $[\mathrm{H}$.

For $u=\left(i_{1}, i_{2}, \cdots, i_{n}\right) \in\{0,1\}^{n}$, set $V_{u}=V_{i_{1}} V_{i_{2}} \cdots V_{i_{n}}$. The elements

$$
V_{u} V_{u}^{*}, \quad u \in \bigcup_{n}\{0,1\}^{n},
$$

generate an abelian $C^{*}$-subalgebra of $O_{2}$ which is isomorphic to $C\left(\{0,1\}^{\mathbb{N}}\right)$. In the following we identify these two algebras. If the self-adjoint $A$ above comes from $C\left(\{0,1\}^{\mathbb{N}}\right)$, the formula (1.1) will define a continuous one-parameter group $\sigma^{A}$. Therefore, when we define $F:\{0,1\}^{\mathbb{N}} \rightarrow \mathbb{R}$ such that

$$
F\left(\left(x_{i}\right)_{i=1}^{\infty}\right)= \begin{cases}\frac{1}{\min \left\{i: x_{i}=0\right\}}, & \left(x_{i}\right)_{i=1}^{\infty} \neq 1^{\infty} \\ 0, & \left(x_{i}\right)_{i=1}^{\infty}=1^{\infty},\end{cases}
$$

where $1^{\infty} \in\{0,1\}^{\mathbb{N}}$ denotes the infinite string of 1 's, we have specified a continuous and real-valued function on $\{0,1\}^{\mathbb{N}}$ and we can consider the corresponding oneparameter group $\sigma^{F}$. Our main result is the following.

Version: March 9, 2022. 
Theorem 1.1. Let $\beta_{0}$ be the positive real number for which

$$
\sum_{k=1}^{\infty} \exp \left(-\beta_{0} \sum_{j=1}^{k} \frac{1}{j}\right)=1 \text {. }
$$

There are no $\beta$-KMS states for $\sigma^{F}$ when $\beta<\beta_{0}$, a unique $\beta_{0}-K M S$ state and for $\beta>\beta_{0}$ the simplex of $\beta-K M S$ states is affinely homeomorphic to the Bauer simplex of Borel probability measures on the circle $\mathbb{T}$.

The factor types of the extremal KMS states are determined in Section 4. They are all of type $I_{\infty}$, except the unique $\beta_{0}$-KMS state which is of type $I I I_{1}$.

Acknowledgement I thank Johannes Christensen for helpful remarks and corrections to the first versions of this paper.

\section{KMS-STATES ON THE $C^{*}$-ALGEBRA OF A LOCAL HOMEOMORPHISM}

The proof of Theorem 1.1 and the following description of the KMS-states is based on the work of Neshveyev, [N], which offers a very general description of the KMSstates for a class of one-parameter groups of automorphisms on the (full) $C^{*}$-algebra of a locally compact Hausdorff étale groupoid. To describe the relevant (and wellknown) groupoid, note that the shift $\sigma$ acts on $\{0,1\}^{\mathbb{N}}$ as a local homeomorphism, given by $\sigma(x)_{i}=x_{i+1}$, and recall that there is a general construction of an étale groupoid from a local homeomorphism which was introduced by Renault in [Re1] in the setting relevant for our main result. Since the work of Renault the construction of the groupoid and the associated $C^{*}$-algebra has been extended by Deaconu [De], by Anantharaman-Delaroche, An, and finally by Renault [Re2 again. We shall now describe the groupoid from $\mathrm{An}$ and explain what information can be obtained by applying Neshveyev's work to it.

Let $X$ be a locally compact second countable Hausdorff space and $\varphi: X \rightarrow X$ a local homeomorphism. Set

$$
\mathcal{G}_{\varphi}=\left\{(x, n-m, y) \in X \times \mathbb{Z} \times X: \varphi^{n}(x)=\varphi^{m}(y)\right\}
$$

which is a groupoid with product $(x, k, y)(y, l, z)=(x, k+l, y)$ and inversion $(x, k, y)^{-1}=$ $(y,-k, x)$. Sets of the form

$$
\left\{(x, n-m, y): \varphi^{n}(x)=\varphi^{m}(y), x \in W, y \in V\right\},
$$

for some open subsets $V, W \subseteq X$ constitute a basis for a topology in $\mathcal{G}_{\varphi}$ which turns it into a locally compact second countable Hausdorff étale groupoid. As explained by Renault in Re2 the groupoid $\mathcal{G}_{\varphi}$ is amenable and hence the full and reduced groupoid $C^{*}$-algebras of $\mathcal{G}_{\varphi}$ coincide, cf. [Re1. We denote this $C^{*}$-algebra by $C^{*}\left(\mathcal{G}_{\varphi}\right)$.

Let $F: X \rightarrow \mathbb{R}$ be a continuous function. We can then define a continuous homomorphism $c_{F}: \mathcal{G}_{\varphi} \rightarrow \mathbb{R}$ such that

$$
c_{F}(x, k, y)=\lim _{n \rightarrow \infty}\left(\sum_{i=0}^{n+k} F\left(\varphi^{i}(x)\right)-\sum_{i=0}^{n} F\left(\varphi^{i}(y)\right)\right) .
$$

There is a one-parameter group $\sigma^{F}$ on $C^{*}\left(\mathcal{G}_{\varphi}\right)$ defined such that

$$
\sigma_{t}^{F}(f)(x, k, y)=e^{i t c_{F}(x, k, y)} f(x, k, y)
$$


when $f \in C_{c}\left(\mathcal{G}_{\varphi}\right)$, cf. [Re1]. Let $\beta \in \mathbb{R}$. To describe the $\beta$-KMS-states of $\sigma^{F}$ we say that a Borel measure $m$ on $X$ is $e^{\beta F}$-conformal for $\varphi$ when

$$
m(\varphi(A))=\int_{A} e^{\beta F(x)} d m(x)
$$

for every Borel subset $A \subseteq X$ such that $\varphi: A \rightarrow X$ is injective. This definition is due to Denker and Urbanski, [DU], motivated by work of D. Sullivan. By Lemma 3.2 in [Th2 the condition is equivalent to $m$ being $\left(\mathcal{G}_{\varphi}, c_{F}\right)$-conformal with exponent $\beta$ as defined in [Th1]. A $e^{\beta F}$-conformal Borel probability measure $m$ on $X$ gives therefore rise to a $\beta$-KMS state $\phi_{m}$ for $\sigma^{F}$ such that

$$
\phi_{m}(f)=\int_{X} f(x, 0, x) d m(x)
$$

when $f \in C_{c}\left(\mathcal{G}_{\varphi}\right)$. The calculation necessary to confirm this was first performed by Renault, cf. Proposition 5.4 in [Re1]. One of the main achievements in [N] is the realization that KMS states of this sort do not exhaust them all.

Let $x \in X$ be a $\varphi$-periodic point such that

$$
\sum_{j=0}^{p-1} F\left(\varphi^{j}(x)\right)=0
$$

when $p$ is the minimal period of $x$. Assume that $\beta \in \mathbb{R}$ is such that

$$
M=\sum_{n=1}^{\infty} \sum_{y \in Y_{n}} \exp \left(-\beta \sum_{j=0}^{n-1} F\left(\varphi^{j}(y)\right)\right)<\infty
$$

where

$$
Y_{n}=\varphi^{-n}(x) \backslash \bigcup_{j=0}^{n-1} \varphi^{-j}(x)
$$

For $y \in X$, let $\delta_{y}$ be the Dirac probability measure at $y$. Then

$$
m_{x}=(1+M)^{-1}\left(\delta_{x}+\sum_{n=1}^{\infty} \sum_{y \in Y_{n}} \exp \left(-\beta \sum_{j=0}^{n-1} F\left(\varphi^{j}(y)\right)\right) \delta_{y}\right)
$$

is a Borel probability measure and it is easy to check, using (2.3), that $m_{x}$ is $e^{\beta F_{-}}$ conformal. For $\lambda \in \mathbb{T}$ and $y \in \bigcup_{n=0}^{\infty} \varphi^{-n}(x)$, the map $(y, k p, y) \mapsto \lambda^{k}$ is a character of the isotropy group

$$
\left(\mathcal{G}_{\varphi}\right)_{y}^{y}=\{(y, k p, y): k \in \mathbb{Z}\} .
$$

Since $m_{x}$ is supported on $\bigcup_{n=0}^{\infty} \varphi^{-n}(x)$ we can consider this as a $m_{x}$-measurable field of states on the isotropy groups of $\mathcal{G}_{\varphi}$ in the sense of [N]. It follows therefore from Theorem 1.1 in [N] that there is a state $\phi_{x}^{\lambda}$ on $C^{*}\left(\mathcal{G}_{\varphi}\right)$ such that

$$
\phi_{x}^{\lambda}(f)=\int_{X} \sum_{k \in \mathbb{Z}} \lambda^{k} f(y, k p, y) d m_{x}(y)
$$

for all $f \in C_{c}\left(\mathcal{G}_{\varphi}\right)$. Furthermore, it follows from Theorem 1.3 in [N] that $\phi_{x}^{\lambda}$ is a $\beta$-KMS state. These facts follow also from the following lemma. To formulate it, set $\Lambda=\bigcup_{n=0}^{\infty} \varphi^{-n}(x)$ and for $y \in \Lambda$ set

$$
|y|=\min \left\{k \in \mathbb{N}: \varphi^{k}(y)=x\right\} .
$$


Define a one-parameter group $U_{t}, t \in \mathbb{R}$, of unitaries on $l^{2}(\Lambda)$ such that

$$
U_{t} \psi(y)=\exp \left(i t \sum_{j=0}^{|y|-1} F\left(\varphi^{j}(y)\right)\right) \psi(y)
$$

and a positive operator $H_{\beta}$ by

$$
H_{\beta} \psi(y)=\exp \left(-\beta \sum_{j=0}^{|y|-1} F\left(\varphi^{j}(y)\right)\right) \psi(y) .
$$

It follows from (2.4) that $H_{\beta}$ is trace-class. Note that $H_{\beta}=U_{i \beta}$.

Lemma 2.1. For each $\lambda \in \mathbb{T}$ there is an irreducible $*$-representation $\pi_{\lambda}$ of $C^{*}\left(\mathcal{G}_{\varphi}\right)$ on $l^{2}(\Lambda)$ such that

$$
\pi_{\lambda} \circ \sigma_{t}^{F}=\operatorname{Ad} U_{t} \circ \pi_{\lambda}
$$

for all $t \in \mathbb{R}$, and

$$
\phi_{x}^{\lambda}(a)=\frac{\operatorname{Tr}\left(H_{\beta} \pi_{\lambda}(a)\right)}{\operatorname{Tr}\left(H_{\beta}\right)}
$$

for all $a \in C^{*}\left(\mathcal{G}_{\varphi}\right)$.

Proof. When $\varphi, \psi \in l^{2}(\Lambda)$ and $f \in C_{c}\left(\mathcal{G}_{\varphi}\right)$ we have the estimate

$$
\begin{aligned}
& \left(\sum_{y, z, k}|\varphi(y)||f(y,|y|-|z|+k p, z)||\psi(z)|\right)^{2} \\
& \leq \sum_{y, z, k}|\varphi(y)|^{2}|f(y,|y|-|z|+k p, z)| \sum_{y^{\prime}, z^{\prime}, k^{\prime}}\left|\psi\left(z^{\prime}\right)\right|^{2}\left|f\left(y^{\prime},\left|y^{\prime}\right|-\left|z^{\prime}\right|+k^{\prime} p, z^{\prime}\right)\right| \\
& \leq\|f\|_{I}^{2} \sum_{y}|\varphi(y)|^{2} \sum_{z}|\psi(z)|^{2}
\end{aligned}
$$

where $\|f\|_{I}$ is the $I$-norm of Renault, cf. [Re1]. Since $\|f\|_{I}<\infty$, it follows that

$$
\pi_{\lambda}(f) \psi(y)=\sum_{z \in \Lambda} \sum_{k \in \mathbb{Z}} \lambda^{k} f(y,|y|-|z|+k p, z) \psi(z)
$$

defines an element $\pi_{\lambda}(f) \psi \in l^{2}(\Lambda)$ when $\psi \in l^{2}(\Lambda)$. Furthermore, we see that $\pi_{\lambda}(f)$ is a bounded operator on $l^{2}(\Lambda)$ with $\left\|\pi_{\lambda}(f)\right\| \leq\|f\|_{I}$. It follows that $\pi_{\lambda}$ extends to a map $\pi_{\lambda}: C^{*}\left(\mathcal{G}_{\varphi}\right) \rightarrow B\left(l^{2}(\Lambda)\right)$. It is straightforward to verify that $\pi_{\lambda}$ is an irreducible $*$-representation with the stated properties. We leave the details with the reader who will then see how condition (2.3) is used to establish (2.7).

It follows that a $p$-periodic point $x$ of $\varphi$ for which (2.3) and (2.4) both hold gives rise to a $\beta$-KMS state $\phi_{x}^{\mu}$ for every Borel probability measure $\mu$ on the circle such that

$$
\phi_{x}^{\mu}=\int_{\mathbb{T}} \phi_{x}^{\lambda} d \mu(\lambda)
$$

In this way such a periodic orbit gives rise to a face in the simplex of $\beta$-KMS states which is affinely homeomorphic to the Bauer simplex of Borel probability measures on the circle. Note that the faces of $\beta$-KMS states coming from different periodic points are the same when the periodic points are in the same orbit and disjoint otherwise. Note also that it follows from Lemma 2.1 that $\varphi_{x}^{\lambda}$ is an extremal 
$\beta$-KMS state, cf. Theorem 5.3.30 (3) in [BR]. If we assume that the $\varphi$-periodic points are countable, and if $\beta \neq 0$, it follows from Theorem 2.4 in [Th1] that the $\beta$-KMS state $\varphi_{m}$ of (2.2) arising from a continuous (or non-atomic) $e^{\beta F}$-conformal Borel probability measure $m$ is extremal if and only of $m$ is extremal in the set of $e^{\beta F}$-conformal Borel probability measures. But note that the 'if'-part is not true for measures with atoms. For example, the measure $m_{x}$ is extremal among the $e^{\beta F}$-conformal measures while

$$
\phi_{m_{x}}=\int_{\mathbb{T}} \phi_{x}^{\lambda} d \lambda
$$

when we integrate with respect to Lebesgue measure $d \lambda$.

We have now described the extremal KMS states that are relevant for the example considered in this paper, but for completeness we describe them all. The full orbit $\mathcal{O}(z)$ of a point $z \in X$ is the set points $y \in X$ such that

$$
\varphi^{n}(z)=\varphi^{m}(y)
$$

for some $n, m \in \mathbb{N}$. An element $z \in X$ is aperiodic when $\mathcal{O}(z)$ does not contain a periodic orbit. For such a point $z$ we can define $\mathcal{F}: \mathcal{O}(z) \rightarrow \mathbb{R}$ such that

$$
\mathcal{F}(y)=\sum_{j=0}^{m-1} F\left(\varphi^{j}(y)\right)-\sum_{j=0}^{n-1} F\left(\varphi^{j}(z)\right),
$$

where $n, m$ are numbers such that (2.8) holds. For $\beta \in \mathbb{R}$ we say that $z$ is $\beta$-summable when

$$
M=\sum_{y \in \mathcal{O}(z)} e^{-\beta \mathcal{F}(y)}<\infty,
$$

and we can then consider the Borel probability measure

$$
m_{z}=M^{-1} \sum_{y \in \mathcal{O}(z)} e^{-\beta \mathcal{F}(y)} \delta_{y} .
$$

This is a $e^{\beta F}$-conformal measure and we can consider the corresponding state $\phi_{m_{z}}$ which is an extremal $\beta$-KMS state. By Theorem 1.3 in [N] and Theorem 2.4 in [Th1] we have now described all extremal $\beta$-KMS states for $\sigma^{F}$ and can summarize with the following

Theorem 2.2. Assume that the periodic points of $\varphi$ are countable. Let $\beta \in \mathbb{R} \backslash\{0\}$. The extremal $\beta$-KMS states for $\sigma^{F}$ are

- the states $\phi_{m}$, where $m$ is an extremal and continuous $e^{\beta F}$-conformal Borel probability measure $m$ on $X$,

- the states $\varphi_{x}^{\lambda}$, where $\lambda \in \mathbb{T}$ and $x$ is a p-periodic point for $\varphi$ for which (2.3) and (2.4) both hold, and

- the states $\phi_{m_{z}}$, where $z \in X$ is aperiodic and $\beta$-summable.

When $\varphi$ is surjective and does not change sign, in the sense that either $F(x) \geq 0$ for all $x \in X$ or $F(x) \leq 0$ for all $x \in X$, no aperiodic point can be $\beta$-summable for any $\beta$, and there are then only the two first-mentioned classes of KMS-states to consider. 


\section{Proof of the MAIN RESUlt}

We apply Theorem 2.2 to the shift $\sigma$ on $\{0,1\}^{\mathbb{N}}$ and the function $F$ defined by (1.3). For this note that there is a $*$-isomorphism $O_{2} \simeq C^{*}\left(\mathcal{G}_{\sigma}\right)$ sending the isometry $V_{i} \in O_{2}$ to the characteristic function of the set $\left\{(x, 1, \sigma(x)) \in \mathcal{G}_{\sigma}: x_{1}=i\right\}, i=0,1$. Under this isomorphism the one-parameter group $\sigma^{F}$ from Section 1 is turned into the one-parameter group $\sigma^{F}$ from Section 2 .

Lemma 3.1. For each $\beta \in \mathbb{R}$ there is at most one $e^{\beta F}$-conformal probability measure for $\sigma$, and none if $\beta<\beta_{0}$.

Proof. For every word $u \in\{0,1\}^{n}$ we let $[u]$ denote the corresponding cylinder set,

$$
[u]=\left\{\left(x_{i}\right)_{i=1}^{\infty} \in\{0,1\}^{\mathbb{N}}: x_{1} x_{2} \cdots x_{n}=u\right\} .
$$

It follows from (2.1) that a $e^{\beta F}$-conformal Borel probability measure $\mu$ must satisfy

$$
e^{\beta} \mu([0])=\mu\left(\{0,1\}^{\mathbb{N}}\right)=1,
$$

and hence $\mu([0])=e^{-\beta}$ and $\mu([1])=1-e^{-\beta}$. Now assume that we have determined $\mu([w])$ for every word $w \in\{0,1\}^{n}$. Let $w$ be such a word. Then

$$
\mu([w])=\int_{[0 w]} e^{\beta F(x)} d \mu(x)=e^{\beta} \mu([0 w]),
$$

and we conclude that $\mu([0 w])=e^{-\beta} \mu([w])$. Assume then that $w$ does not consist entirely of 1 's, and let $j$ be position of the first 0 occurring in $w$. Then

$$
\mu([w])=\int_{[1 w]} e^{\beta F(x)} d \mu(x)=e^{\frac{\beta}{j+1}} \mu([1 w]),
$$

and hence $\mu([1 w])=e^{-\frac{\beta}{j+1}} \mu([w])$. In this way we determine the value $\mu([u])$ for every word $u \in\{0,1\}^{n+1}$, except the word consisting entirely of 1 's. However, that number is then determined by the condition that

$$
\sum_{u \in\{0,1\}^{n+1}} \mu([u])=1
$$

This proves the uniqueness of a $e^{\beta F}$-conformal Borel probability measure since regular Borel measures are determined by the values they give to cylinder sets.

To prove that there can not be any $e^{\beta F}$-conformal probability measure unless $\beta \geq \beta_{0}$, observe that the sets $[0],[10],[110],[1110], \cdots$, are mutually disjoint, and that for any Borel measure $\mu$ satisfying (2.1) we have

$$
\mu\left(\left[1^{k-1} 0\right]\right)=\exp \left(-\beta \sum_{j=1}^{k} \frac{1}{j}\right) \mu\left(\{0,1\}^{\mathbb{N}}\right) .
$$

If $\mu$ is a probability measure we must therefore have that $\sum_{k=1}^{\infty} \exp \left(-\beta \sum_{j=1}^{k} \frac{1}{j}\right) \leq$ 1, i.e. $\beta \geq \beta_{0}$.

Lemma 3.2. Let $\beta \in \mathbb{R}$. Then

$$
\sum_{n=1}^{\infty} \sum_{x \in \sigma^{-n}\left(1^{\infty}\right) \backslash \sigma^{-n+1}\left(1^{\infty}\right)} \exp \left(-\beta \sum_{j=0}^{n-1} F\left(\sigma^{j}(x)\right)\right)<\infty
$$


if and only if $\beta>\beta_{0}$.

Proof. Set $Y_{0}=\left\{1^{\infty}\right\}$ and $Y_{n}=\sigma^{-n}\left(1^{\infty}\right) \backslash \sigma^{-n+1}\left(1^{\infty}\right), n \geq 1$. Then

$$
Y_{n}=0 Y_{n-1} \sqcup 10 Y_{n-2} \sqcup 110 Y_{n-3} \sqcup 1110 Y_{n-4} \sqcup \cdots \sqcup 1^{n-1} 0 Y_{0} .
$$

Set

$$
Z_{n}=\sum_{x \in Y_{n}} \exp \left(-\beta \sum_{j=0}^{n} F\left(\sigma^{j}(x)\right)\right),
$$

for $n \geq 0$, and $s_{k}=1+\frac{1}{2}+\frac{1}{3}+\cdots+\frac{1}{k}$ for $k \geq 1$. It follows from (3.1) that

$$
Z_{n}=e^{-\beta s_{1}} Z_{n-1}+e^{-\beta s_{2}} Z_{n-2}+\cdots+e^{-\beta s_{n}} Z_{0}
$$

for all $n \geq 1$, and then from (3.2) that

$$
\sum_{n=1}^{N} Z_{n} \leq\left(\sum_{n=0}^{N} Z_{n}\right)\left(\sum_{k=1}^{N} e^{-\beta s_{k}}\right) \leq \sum_{n=1}^{2 N} Z_{n}
$$

for all $N \geq 1$. It is straightforward to deduce from (3.3) that $\sum_{k=1}^{\infty} e^{-\beta s_{k}}<1$ if and only if $\sum_{n=1}^{\infty} Z_{n}<\infty$. This completes the proof.

Except for the existence of a $\beta_{0}$-KMS state, Theorem 1.1 follows now by combining Lemma 3.1 and Lemma 3.2 with Theorem 2.2. That there is a $\beta_{0}$-KMS state follows from the fact that set of $\beta$ 's for which there is a $\beta$-KMS state is closed by Proposition 5.3.25 in [BR].

\section{FACTOR TYPES}

Theorem 4.1. For $\beta>\beta_{0}$ the von Neumann algebra factor generated by the GNS representation of an extremal $\beta-K M S$ state for $\sigma^{F}$ is of type $I_{\infty}$. The von Neumann algebra generated by the GNS representation of the unique $\beta_{0}-K M S$ state for $\sigma^{F}$ is the hyper-finite III $I_{1}$-factor.

Proof. The assertion concerning the cases when $\beta>\beta_{0}$ follows from Lemma 2.1 . Let $\omega$ be the $\beta_{0}$-KMS state. It follows from Theorem 2.2 and Lemma 3.2 that there is a continuous Borel probability measure $\mu$ on $\{0,1\}^{\mathbb{N}}$ such that

$$
\omega(f)=\int_{\{0,1\}^{\mathbb{N}}} f(x, 0, x) d \mu(x)
$$

for $f \in C_{c}\left(\mathcal{G}_{\sigma}\right)$. Let $\pi_{\omega}$ be the GNS-representation of $\omega$ and set $M=\pi_{\omega}\left(C^{*}\left(\mathcal{G}_{\sigma}\right)\right)^{\prime \prime}$. Then $M$ is a $\sigma$-finite injective factor since $C^{*}\left(\mathcal{G}_{\sigma}\right)$ is separable and nuclear, and $\omega$ is an extremal $\beta_{0}$-KMS state. By Haagerups result, [Ha], it suffices therefore now to show that $\Gamma(M)=\mathbb{R}$ where $\Gamma(M)$ is the invariant from Connes' thesis, $\mathrm{C}$. This will be achieved via an elaboration of the method used for the proof of Proposition 4.11 in [Th2] and Theorem 3.2 in [Th3]. Let $\tilde{\omega}$ be the faithful normal state on $M$ extending $\omega$ in the sense that $\tilde{\omega} \circ \pi_{\omega}=\omega$. The modular automorphism group $\theta$ of $M$ corresponding to $\tilde{\omega}$ is $\theta_{t}=\tilde{\sigma}_{\beta_{0} t}^{F}$, where $\tilde{\sigma}^{F}$ is the $\sigma$-weakly continuous one-parameter group such that $\tilde{\sigma}_{t}^{F} \circ \pi_{\omega}=\pi_{\omega} \circ \sigma_{t}^{F}$ for all $t$, cf. Theorem 8.14.5 in [Pe]. It follows from [C] that

$$
\Gamma(M)=\bigcap_{q} \operatorname{Sp}(q M q)
$$


where we take the intersection over all non-zero central projections $q$ in the fixed point algebra $N$ of $\theta$, and $\operatorname{Sp}(q M q)$ is the Arveson spectrum of the restriction of $\theta$ to $q M q$. Since $\Gamma(M)$ is a closed subgroup of $\mathbb{R}$ it suffices to take a $K \in \mathbb{N}, K \geq 2$, and a non-zero central projection $q$ in $N$ and show that

$$
\frac{\beta_{0}}{K+1} \in \operatorname{Sp}(q M q) \text {. }
$$

In the following we identify $\{0,1\}^{\mathbb{N}}$ with the unit space of $\mathcal{G}_{\sigma}$ via the embedding $x \mapsto(x, 0, x) \in \mathcal{G}_{\sigma}$, and we set $\|a\|_{\omega}=\sqrt{\tilde{\omega}\left(a^{*} a\right)}$ for $a \in M$. Furthermore, to simplify notation we suppress $\pi_{\omega}$ from the notation and consider $C_{c}\left(\mathcal{G}_{\sigma}\right)$ as a subalgebra of $M$. Finally, we use $r$ and $s$ to denote the range and source map of $\mathcal{G}_{\sigma}$, i.e. $r(x, k, y)=x$ and $s(x, k, y)=y$.

To approximate $q$ with elements from $C\left(\{0,1\}^{\mathbb{N}}\right)$ we first prove

Assertion 4.2. For every $f \in C_{c}\left(\mathcal{G}_{\sigma}\right)$ and every $\epsilon>0$ there is a finite set $d_{j}, j=$ $0,1,2, \cdots, m$, of elements in $C\left(\{0,1\}^{\mathbb{N}}\right)$ such that $\sum_{j=0}^{m} d_{j}^{2}=1$ and $\sum_{j=0}^{m} d_{j} f d_{j}=$ $\left.f\right|_{\{0,1\}^{\mathbb{N}}}+h$ where $\|h\|_{\omega} \leq \epsilon$.

Set Is $\mathcal{G}_{\sigma}=\left\{(x, k, x) \in \mathcal{G}_{\sigma}: x \in\{0,1\}^{\mathbb{N}}, k \neq 0\right\}$. Write $f=\left.f\right|_{\{0,1\}^{\mathbb{N}}}+f_{1}$ where supp $f_{1} \subseteq \mathcal{G}_{\sigma} \backslash\{0,1\}^{\mathbb{N}}$. Consider an open bisection in $\mathcal{G}_{\sigma}$ of the form

$$
\mathcal{U}=\left\{(x, n-m, y): \sigma^{n}(x)=\sigma^{m}(y), x \in W, y \in V\right\}
$$

with $\sigma^{n}$ injective on $W$ and $\sigma^{m}$ injective on $V$, and $n \neq m$. An element $\mathcal{U} \cap$ Is $\mathcal{G}_{\sigma}$ must have the form $(z, n-m, z)$ where $\sigma^{\min \{n, m\}}(z)$ is $|n-m|$-periodic for $\sigma$. There are only finitely many such elements $z$ and we conclude therefore that $\operatorname{supp} f_{1} \cap$ Is $\mathcal{G}_{\sigma}$ is a finite set. Since $\mu$ is continuous, $\mu\left(r\left(\operatorname{supp} f_{1} \cap\right.\right.$ Is $\left.\left._{\mathcal{G}_{\sigma}}\right)\right)=0$ and we can choose an open neighborhood $U$ of $r\left(\operatorname{supp} f_{1} \cap\right.$ Is $\left.\mathcal{G}_{\sigma}\right)$ such that

$$
\mu(U) \leq \frac{\epsilon^{2}}{4\|f\|^{2}+1}
$$

Write $f_{1}=f_{2}+f_{3}$ where $f_{2} \in C_{c}\left(r^{-1}(U) \cap s^{-1}(U)\right)$ and supp $f_{3} \subseteq \mathcal{G}_{\sigma} \backslash\left(\{0,1\}^{\mathbb{N}} \cup\right.$ Is $\left.\mathcal{G}_{\sigma}\right)$. For each $x \in r\left(\operatorname{supp} f_{3}\right)$ there is an open neighborhood $U_{x}$ of $x$ such that

$$
s\left(r^{-1}\left(U_{x}\right) \cap \operatorname{supp} f_{3}\right) \cap U_{x}=\emptyset .
$$

It follows that there is a finite open cover $U_{i}, i=1,2, \cdots, m$, of $r\left(\operatorname{supp} f_{3}\right)$ such that $s\left(r^{-1}\left(U_{i}\right) \cap \operatorname{supp} f_{3}\right) \cap U_{i}=\emptyset$ for each $i$. Let $k_{j}, j=0,1,2, \cdots, m$, be a partition of unity in $C\left(\{0,1\}^{\mathbb{N}}\right)$ such that $\operatorname{supp} k_{j} \subseteq U_{j}, j=1,2, \cdots, m$, and $\operatorname{supp} k_{0} \cap$ $r\left(\operatorname{supp} f_{3}\right)=\emptyset$. Set $d_{j}=\sqrt{k_{j}}$. Then $\sum_{j=0}^{m} d_{j} f_{3} d_{j}=0$ and hence

$$
\sum_{j=0}^{m} d_{j} f d_{j}=\left.f\right|_{\{0,1\}^{\mathbb{N}}}+\sum_{j=0}^{m} d_{j} f_{2} d_{j} .
$$

Set $h=\sum_{j=0}^{m} d_{j} f_{2} d_{j}$. Then $\|h\| \leq 2\|f\|$ and $\operatorname{supp} h \subseteq r^{-1}(U) \cap s^{-1}(U)$. In particular, from the last fact it follows that $\left.h^{*} h\right|_{\{0,1\}^{\mathbb{N}}}$ is supported in $U$ and we find therefore from (4.2) that

$$
\|h\|_{\omega}^{2}=\left.\int_{\{0,1\}^{\mathbb{N}}} h^{*} h\right|_{\{0,1\}^{\mathbb{N}}} d \mu \leq\|h\|^{2} \mu(U) \leq \epsilon^{2} .
$$

This proves Assertion 4.2. To use it, note that an application of Kaplansky's density theorem gives us a sequence $\left\{g_{n}\right\}$ of self-adjoint elements in $C_{c}\left(\mathcal{G}_{\sigma}\right)$ such that $\left\|g_{n}\right\| \leq$ 
1 for all $n$ and $\lim _{n \rightarrow \infty}\left\|q-g_{n}\right\|_{\omega}=0$. Set $f_{n}=\left.g_{n}\right|_{\{0,1\}^{\mathbb{N}}}$ and let $\epsilon>0$. Let $\left\{d_{j}\right\}_{j=0}^{m}$ be a partition of unity in $C\left(\{0,1\}^{\mathbb{N}}\right)$ obtained by applying Assertion 4.2 with $f=g_{n}$. Using Kadisons Schwarz-inequality for the second inequality and the KMS-property for the second equality we find that

$$
\begin{aligned}
& \left\|q-f_{n}\right\|_{\omega}-\epsilon \leq\left\|q-\sum_{j=0}^{m} d_{j} g_{n} d_{j}\right\|_{\omega}=\left\|\sum_{j=0}^{m} d_{j}\left(q-g_{n}\right) d_{j}\right\|_{\omega} \\
& \leq \sqrt{\tilde{\omega}\left(\sum_{j=0}^{m} d_{j}\left(q-g_{n}\right)^{2} d_{j}\right)}=\sqrt{\tilde{\omega}\left(\sum_{j=0}^{m}\left(q-g_{n}\right)^{2} d_{j}^{2}\right)}=\left\|q-g_{n}\right\|_{\omega} .
\end{aligned}
$$

It follows that $\lim _{n \rightarrow \infty}\left\|q-f_{n}\right\|_{\omega}=\lim _{n \rightarrow \infty}\left\|q-g_{n}\right\|_{\omega}=0$. Since finite linear combinations of $\left\{1_{[u]}: u \in \bigcup_{n}\{0,1\}^{n}\right\}$ is a norm-dense *-subalgebra of $C\left(\{0,1\}^{\mathbb{N}}\right)$ we may assume that each $g_{n}$ is such a linear combination. Since $q$ is a projection a standard argument, as in the proof of Lemma 12.2.3 in [KR], shows that for every $\epsilon>0$ there is a finite collection $u_{1}, u_{2}, \cdots, u_{L} \in \bigcup_{n}\{0,1\}^{n}$ such that $\left[u_{i}\right] \cap\left[u_{j}\right]=\emptyset$ when $i \neq j$, and

$$
\|q-p\|_{\omega}<\epsilon
$$

where $\epsilon>0$ is as small as we need, and $p=\sum_{i=1}^{L} 1_{\left[u_{i}\right]}$. We choose $\epsilon>0$ so small that

$$
\exp \left(-\beta_{0}\left(\sum_{j=1}^{K} \frac{1}{j}\right)\right) \epsilon+\exp \left(\frac{\beta_{0}}{K}\right) \epsilon+2 \epsilon<\exp \left(-\beta_{0}\left(\sum_{j=1}^{K+1} \frac{1}{j}\right)\right) \tilde{\omega}(q) .
$$

Fix an $i$ and consider the mutually disjoint sets $\left[u_{i} 0\right],\left[u_{i} 10\right],\left[u_{i} 110\right],\left[u_{i} 1110\right], \cdots$. Since $\left[u_{i}\right] \backslash \bigcup_{j=0}^{\infty}\left[u_{i} 1^{j} 0\right]$ only contains one element, and since $\mu$ does not have atoms, we conclude that

$$
\lim _{m \rightarrow \infty}\left\|1_{\left[u_{i}\right]}-\sum_{j=0}^{m} 1_{\left[u_{i} 1^{j} 0\right]}\right\|_{\omega}=0 .
$$

Exchanging $u_{i}$ with $u_{i} 1^{j} 0, j=0,1,2, \cdots, n$, for some sufficiently large $n$, and doing a similar thing with the other $u_{i}$ 's, we can arrange that each $u_{i}$ terminates with 0 . Set $l^{+}=1^{K-1} 0, l^{-}=1^{K} 0$ and $\left|l^{+}\right|=K,\left|l^{-}\right|=K+1$. For each $i$ we let $w_{i}^{ \pm} \in C_{c}\left(\mathcal{G}_{\sigma}\right)$ be the characteristic function of the compact and open set

$$
\left\{\left(u_{i} x,-\left|l^{ \pm}\right|, u_{i} l^{ \pm} x\right): x \in\{0,1\}^{\mathbb{N}}\right\}
$$

in $\mathcal{G}_{\sigma}$. Each $w_{i}^{ \pm}$is a partial isometry such that

a) $w_{i}^{ \pm} w_{i}^{ \pm *}=1_{\left[u_{i}\right]}$,

b) $w_{i}^{ \pm *} w_{i}^{ \pm}=1_{\left[u_{i} l^{ \pm}\right]} \leq 1_{\left[u_{i}\right]}$, and

c) $\sigma_{t}^{F}\left(w_{i}^{+}\right)=\exp \left(-i t\left(\sum_{j=1}^{K} \frac{1}{j}\right)\right) w_{i}^{+}$while $\sigma_{t}^{F}\left(w_{i}^{-}\right)=\exp \left(-i t\left(\sum_{j=1}^{K+1} \frac{1}{j}\right)\right) w_{i}^{-}$ for all $t \in \mathbb{R}$.

By using that $u_{i}$ terminates with 0 , and that $l^{-}=1^{K} 0$, it follows from the conformality condition (2.1) and the definition of $F$ that

$$
\mu\left(\left[u_{i} l^{-}\right]\right)=\exp \left(-\beta_{0}\left(\sum_{j=1}^{K+1} \frac{1}{j}\right)\right) \mu\left(\left[u_{i}\right]\right) .
$$


Set $w_{ \pm}=\sum_{i=1}^{L} w_{i}^{ \pm}$and note that $w_{ \pm}$are partial isometries. It follows from $\mathrm{b}$ ) that $w_{+} p=w_{+}$and therefore from (4.3) that

$$
\tilde{\omega}\left(q w_{+}{ }^{*} w_{-} q w_{-}{ }^{*} w_{+} q\right) \geq \tilde{\omega}\left(w_{+}{ }^{*} w_{-} q w_{-}{ }^{*} w_{+}\right)-2 \epsilon .
$$

Since $\tilde{\omega}$ is a $\beta_{0}$-KMS state for $\tilde{\sigma}^{F}$, it follows from c) that

$$
\tilde{\omega}\left(w_{+}{ }^{*} w_{-} q w_{-}{ }^{*} w_{+}\right)=e^{\frac{\beta_{0}}{K+1}} \tilde{\omega}\left(q w_{-}{ }^{*} w_{+} w_{+}{ }^{*} w_{-}\right),
$$

which thanks to a) is the same as $e^{\frac{\beta_{0}}{K+1}} \tilde{\omega}\left(q w_{-}{ }^{*} w_{-}\right)$. Using (4.3) again we find that

$$
\tilde{\omega}\left(q w_{-}^{*} w_{-}\right) \geq \tilde{\omega}\left(p w_{-}^{*} w_{-}\right)-\epsilon .
$$

It follows from b) that $\tilde{\omega}\left(p w_{-}{ }^{*} w_{-}\right)=\tilde{\omega}\left(w_{-}{ }^{*} w_{-}\right)$while b), a) and (4.4) imply that

$$
\tilde{\omega}\left(w_{-}{ }^{*} w_{-}\right)=\mu\left(\cup_{i}\left[u_{i} l^{-}\right]\right)=\exp \left(-\beta_{0}\left(\sum_{j=1}^{K+1} \frac{1}{j}\right)\right) \tilde{\omega}(p) .
$$

Since $\tilde{\omega}(p) \geq \tilde{\omega}(q)-\epsilon$ by (4.3) we can put everything together and conclude that

$$
\begin{aligned}
& \tilde{\omega}\left(q w_{+}{ }^{*} w_{-} q w_{-}{ }^{*} w_{+} q\right) \geq \\
& \exp \left(-\beta_{0}\left(\sum_{j=1}^{K} \frac{1}{j}\right)\right) \tilde{\omega}(q)-\exp \left(-\beta_{0}\left(\sum_{j=1}^{K} \frac{1}{j}\right)\right) \epsilon-\exp \left(\frac{\beta_{0}}{K+1}\right) \epsilon-2 \epsilon .
\end{aligned}
$$

It follows therefore from the choice of $\epsilon$ that $\tilde{\omega}\left(q w_{+}{ }^{*} w_{-} q w_{-}{ }^{*} w_{+} q\right)>0$. By c),

$$
\tilde{\sigma}_{t}^{F}\left(q w_{+}{ }^{*} w_{-} q\right)=e^{-i t \frac{1}{K+1}} q w_{+}{ }^{*} w_{-} q
$$

and hence $\theta_{t}\left(q w_{+}{ }^{*} w_{-} q\right)=e^{-i t \frac{\beta_{0}}{K+1}} q w_{+}{ }^{*} w_{-} q$ for all $t \in \mathbb{R}$. Since $q w_{+}{ }^{*} w_{-} q \neq 0$ we obtain the desired conclusion (4.1) from Lemme 2.3.6 in [C].

\section{REFERENCES}

[An] C. Anantharaman-Delaroche, Purely infinite $C^{*}$-algebras arising from dynamical systems, Bull. Soc. Math. France 125 (1997), 199-225.

[BR] O. Bratteli and D.W. Robinson, Operator Algebras and Quantum Statistical Mechanics $I+I I$, Texts and Monographs in Physics, Springer Verlag, New York, Heidelberg, Berlin, 1979 and 1981.

[C] A. Connes, Une classification des facteurs de type III, Ann. Sci. Ecole Norm. Sup. 6 (1973), 133-252.

[Cu] J. Cuntz, Simple $C^{*}$-algebras generated by isometries, Comm. Math. Phys. 57 (1977), 173-185.

[De] V. Deaconu, Groupoids associated with endomorphisms, Trans. Amer. Math. Soc. 347 (1995), 1779-1786.

[DU] M. Denker and M. Urbanski, On the Existence of Conformal Measures, Trans. Amer. Math. Soc. 328 (1991), 563-587.

[E] R. Exel, KMS states for generalized gauge actions on Cuntz-Krieger algebras (an application of the Ruelle-Perron-Frobenius theorem), Bull. Braz. Math. Soc. (N.S) 35 (2004), 1-12.

[Ha] U. Haagerup, Connes' bicentralizer problem and uniqueness of the injective factor of type $I I I_{1}$, Acta Math. 158 (1987), 95-148.

[H] F. Hofbauer, Examples for the non-uniqueness of the equilibrium state, Trans. Amer. Math. Soc. 228 (1977), 223-241.

[KR] R.V. Kadison and J.R. Ringrose, Fundamentals of the Theory of Operator Algebras II, Academic Press, London 1986. 
[N] S. Neshveyev, KMS states on the $C^{*}$-algebras of non-principal groupoids, J. Operator Theory 70 (2013), 513-530.

[OP] D. Olesen and G.K. Pedersen, Some $C^{*}$-dynamical systems with a single KMS-state, Math. Scand. 42 (1978), 111-118.

[Pe] G. K. Pedersen, $C^{*}$-algebras and their automorphism groups, Academic Press, London, 1979.

[Re1] J. Renault, A Groupoid Approach to $C^{*}$-algebras, LNM 793, Springer Verlag, Berlin, Heidelberg, New York, 1980.

[Re2] J. Renault, Cuntz-like algebras, Operator theoretical methods (Timisoara, 1998), 371386, Theta Found., Bucharest, 2000.

[Th1] K. Thomsen, KMS-states and conformal measures, Comm. Math. Phys. 316 (2012), 615-640. DOI: $10.1007 / \mathrm{s} 00220-012-1591-\mathrm{z}$

[Th2] K. Thomsen, KMS weights on groupoid and graph $C^{*}$-algebras, J. Func. Analysis 266 (2014), 2959-2988.

[Th3] K. Thomsen, KMS weights on graph $C^{*}$-algebras II. Factor types and ground states, arXiv:1412.6762

E-mail address: matkt@math.au.dk

Institut for Matematik, Aarhus University, Ny Munkegade, 8000 Aarhus C, DenMARK 